\title{
Commentary on 'Maternal long-chain PUFA supplementation during protein deficiency improves brain fatty acid accretion in rat pups by altering the milk fatty acid composition of the dam' by Ranade and Rao
}

Perinatal PUFA nutrition is usually studied in the context of resource-replete populations in Western countries. Under conditions of ample dietary energy and protein, study findings may be neutral because long-chain PUFA (LC-PUFA) effects are obscured by high-performing control groups, widerthan-assumed variability and other factors. Moreover, studies of hidden hunger, defined by adequate energy and protein, usually focus on vitamin $\mathrm{A}, \mathrm{Fe}$, or general micronutrients and ignore essential fats. As industrialising nations such as India shift away from traditional whole foods that deliver a net balance of essential fats towards industrially produced linoleic acid-rich oils, often in the name of cholesterol-lowering, reductions in neural tissue $n-3$ DHA are sure to follow, along with higher nutritional demands for preformed DHA. Despite the evidence-based medicine mantra that considers animal data to be inadequate to set dietary guidelines, animal studies can, and in many cases do, represent the highest level of evidence, particularly in the science of nutrition. Consider that no ethical health researcher or institutional review board would ever consider randomising pregnant women to a diet that delivers all fat from linoleate-replete, linolenate-deficient, seed oils (e.g. conventional sunflower, safflower and peanut), definitively shown in animal studies to compromise neural development of offspring, and probably the mental health of the mother; not a single human trial exists on this point, and in light of the animal evidence, it should never be done. Similar situations exist for most of the dietary essential nutrients.

In the Journal of Nutritional Science, Ranade \& Rao ${ }^{(1)}$ present an animal study of importance for nutritional situations in which protein is in short supply. They consider whether preformed DHA and arachidonic acid (ARA) in the dam's diet modify the effects of a low-protein diet on the DHA status of the rat pup brain. Tissue DHA itself is unequivocally known to be required for proper perinatal brain and retinal development and function from many human studies, and probably also in many other life stages. Ranade and Rao show that DHA and ARA provided to rat dams fed $9 \%$ protein diets restore and enhance brain DHA levels in nursing pups at $14 \mathrm{~d}$ compared with a $9 \%$ protein diet devoid of LCPUFA as well as a protein-replete (18\%) LC-PUFA-devoid control group. They also show that rat dam milk DHA and rat pup DHA are the most highly correlated among fatty acids at day 14 of lactation. At one level, these data suggest that limited protein also limits the conversion of precursor $\alpha$-linolenic acid to DHA, at least against a background soyabean oil diet.

These data have important implications for the nutriture of protein-restricted lactating women. They confirm that milk DHA is lower in dams fed low-protein diets, thus suggesting one mechanism by which low protein may adversely affect brain development. They also imply that compromised brain DHA can be restored by provision of preformed DHA. The control diet, American Institute of Nutrition (AIN)-93, derives all fat from commodity soyabean oil in which linoleic acid is more than $50 \%$ of energy from fat, similar to diets of populations consuming high levels of industrially produced seed oils. This is, therefore, a study of linoleic acid-surfeit diets that in general compromise tissue $n$-3 LC-PUFA and inhibit the accumulation of DHA from dietary precursors ${ }^{(2)}$. Ranade $\& \mathrm{RaO}^{(1)}$ show that low protein further restricts brain DHA accretion. Preformed DHA as provided here is known in other contexts, and convincingly in this study, to restore and enhance brain DHA levels. Greater brain DHA is found for human breast-fed infants compared with infants fed LC-PUFA-free formulae, and in 
a plethora of animal studies DHA is known to be required for proper neural function ${ }^{(3)}$. It is therefore likely that the present results apply to human subjects.

Lactovegetarian pregnant and lactating women in India who shift their intake to manufactured linoleate-rich seed oils such as conventional sunflower, safflower, peanut, or soyabean oils are likely to be particularly vulnerable to a DHA deficiency. Plant foods in general have no LC-PUFA, and milk fat has only small amounts of EPA and no DHA. They would be well advised to restrict intake of these oils or increase intake of acceptable vegetarian sources of DHA. The present paper indicates that this step may be of particular importance in protein-limited populations.

There are no conflicts of interest.

\section{J. T. Brenna}

Division of Nutritional Sciences, Cornell University, Ithaca, NY, USA email jtb4@cornell.edu

\section{References}

1. Ranade PS \& Rao SS (2013) Maternal long-chain PUFA supplementation during protein deficiency improves brain fatty acid accretion in rat pups by altering the milk fatty acid composition of the dam. J Nutr Sci 2, 1-13.

2. Brenna JT, Salem N Jr, Sinclair AJ, et al. (2009) alpha-Linolenic acid supplementation and conversion to $n-3$ long-chain polyunsaturated fatty acids in humans. Prostaglandins Leukot Essent Fatty Acids 80, 85-91.

3. Brenna JT (2011) Animal studies of the functional consequences of suboptimal polyunsaturated fatty acid status during pregnancy, lactation and early post-natal life. Matern Child Nutr 7, Suppl. 2, 59-79. 